\title{
Neonatal Cord Tox Panel and Maternal Perinatal Fentanyl Exposure: A Retrospective Chart Review
}

\author{
Joseph Jones', Donna Coy¹, Ryan Mitacek², Stephanie Thompson², Stefan Maxwell2,3 \\ ${ }^{1}$ United States Drug Testing Laboratories, Des Plaines, Illinois, USA \\ ${ }^{2}$ Charleston Area Medical Center, Charleston, West Virginia, USA \\ ${ }^{3}$ Pediatrix Medical Group, Sunrise, Florida, USA \\ Email: joe.jones@usdtl.com
}

How to cite this paper: Jones, J., Coy, D., Mitacek, R., Thompson, S. and Maxwell, S. (2021) Neonatal Cord Tox Panel and Maternal Perinatal Fentanyl Exposure: A Retrospective Chart Review. American Journal of Analytical Chemistry, 12, 324-331. https://doi.org/10.4236/ajac.2021.129020

Received: August 24, 2021

Accepted: September 23, 2021

Published: September 26, 2021

Copyright (c) 2021 by author(s) and Scientific Research Publishing Inc. This work is licensed under the Creative Commons Attribution International License (CC BY 4.0).

http://creativecommons.org/licenses/by/4.0/

\begin{abstract}
Objective: The specific aim of this study was to determine if the currently available cutoff for fentanyl in umbilical cord (UC) was appropriate to distinguish illicit fentanyl exposure from therapeutic in-hospital administration of fentanyl. Study Design: Medical record review was conducted for perinatal administration of fentanyl and the detection of fentanyl in the corresponding routine UC toxicology. Specimens were initially tested with immunoassay followed by mass spectrometry $(n=62)$. Result: Excluding a single specimen that was confirmed positive, specimens were below the assays' limit of quantification. The immunoassay's mean $\mathrm{b} / \mathrm{b}_{0}$ for the cases that received and did not receive fentanyl prior to delivery was $91.3 \% \pm 10.6 \%$ and $98.2 \% \pm 6.5 \%$, respectively $(p=0.003)$. Conclusion: We demonstrated that $U C$ is a suitable specimen type for the detection of fentanyl and that the cutoff selected adequately identifies illicit fentanyl use while not flagging cases where fentanyl was administered by the hospital prior to birth.
\end{abstract}

\section{Keywords}

Fentanyl, Norfentanyl, Umbilical Cord, Prenatal Fentanyl Exposure

\section{Introduction}

Neonatal abstinence syndrome (NAS) occurs in newborns whose mothers used a neuroactive (affecting brain and nervous tissue) substance such as opioids while pregnant [1] [2]. The rate of NAS has been growing due to the increasing use of opioids in recent years [3] [4]. Fentanyl is a common pain medicine used in the obstetric management of labor [5]. Chronic exposure to fentanyl prior to birth 
can potentially result in NAS [6]. Fentanyl can either be used illicitly, abused, or clinically to control chronic pain or pain during labor [7].

Umbilical cord presents a unique opportunity for drug testing and potential identification of NAS [8] [9] [10]. It is available immediately after birth and each birth produces sufficient quantities of specimen for analysis, unlike other specimen types routinely used for newborn toxicology. As a newborn toxicology specimen, umbilical cord analysis can identify maternal drug use during approximately the third trimester of pregnancy [10].

There are limited options for commercial assays to test for in utero fentanyl exposure. In addition, data to guide the selection of an appropriate cut-off for umbilical cord fentanyl detection to distinguish illicit substance exposure from therapeutic in-hospital administration of fentanyl is currently lacking. The specific aim of this study was to determine if the currently available cutoffs for fentanyl in umbilical cord $(500 \mathrm{pg} / \mathrm{g})$ distinguishes illicit fentanyl exposure from therapeutic in-hospital administration of fentanyl.

\section{Methods}

\subsection{Experimental Design}

A secondary analysis of the medical records and umbilical cord toxicology results of births occurring at Charleston Area Medical Center Women and Children's Hospital (CAMC), Charleston, West Virginia, between October 1, 2018 and November 15, 2018 were analyzed for perinatal administration of fentanyl and the detection of fentanyl in the corresponding umbilical cord specimens. Umbilical cord tissue segments were collected according to standard operating procedures (https://www.usdtl.com/) and shipped to United States Drug Testing Laboratories (Des Plaines, IL) for standard of care toxicology testing that included fentanyl. For the study, all specimens received fentanyl confirmation testing via liquid Chromatography with tandem mass spectrometry (LCMSMS). The study was approved by the CAMC Institutional Review Board (IRB Number 19-634).

\subsection{Medical Records Query}

Medical records were queried for perinatal maternal fentanyl administration including the timing, dosage, and duration of fentanyl use. Birth mothers' medical record numbers (MRN) were not extracted. Neonates without linked maternal records were excluded. Cases where fentanyl was found in the umbilical cord without corresponding medical records of fentanyl administration at the hospital or outpatient prescription were considered to be the result of illicit fentanyl use. Data use agreements were obtained prior to starting the study.

\subsection{Materials}

Fentanyl, fentanyl- $d_{5}$, norfentanyl, and norfentanyl- $d_{5}$ were purchased from Cerilliant (Round Rock, TX, USA) as a $100 \mu \mathrm{g} / \mathrm{mL}$ certified reference standard in 
methanol. Solid phase extraction (SPE) columns (CSDAU020 Clean Screen extraction columns; $200 \mathrm{mg} / 10 \mathrm{~mL}$ ) were purchased from United Chemical Technologies (Bristol, PA, USA). Acetonitrile was high performance liquid chromatography (HPLC) grade, and all other reagents were American Chemical Society (ACS) grade or equivalent from Fischer Scientific (Hanover Park, IL, USA).

\subsection{Analytical Methods}

Umbilical segments approximately $10 \mathrm{~cm}$ long was obtained at the time of birth. Cords were stripped of blood, lightly rinsed in sterile saline, placed in sterile plastic specimen containers, and then frozen for subsequent shipment to USDTL. Preliminary testing was performed on day of receipt into the laboratory and analyzed by enzyme-linked immunosorbent assay (Fentanyl ELISA kit, Immunalysis Corporation, Pomona, CA) which is a competitive inhibition immunoassay. Umbilical cord samples $(0.5 \mathrm{~g})$ were homogenized in $3 \mathrm{~mL}$ of acetone and centrifuged. The supernatant was filtered and evaporated to dryness under a stream of nitrogen at $60^{\circ} \mathrm{C}$. The extracts were reconstituted in $700 \mu \mathrm{L}$ of buffer supplied by the immunoassay vendor. Manufacturer instructions were followed for ELISA testing.

The assay was developed and validated as a qualitative test due to its narrow linear range. Results for the immunoassay were reported as $b / b_{0}$ which is the ratio of the specimen's optical density response and optical density response of the negative control. The immunoassay's selected cutoff for fentanyl detection was the mean $\mathrm{b} / \mathrm{b}_{0}$ for three single point calibrators fortified with $500 \mathrm{pg} / \mathrm{g}$ of fentanyl.

For confirmatory analysis, the internal standards (a mixture containing fentanyl- $d_{5}$ and norfentanyl- $\left.d_{5}, 1 \mathrm{ng} / \mathrm{g}\right)$ were added to the umbilical cord $(0.5 \mathrm{~g})$ aliquots and the samples were homogenized in $3 \mathrm{~mL}$ of acetonitrile. Following centrifugation, the supernatants were evaporated to dryness under a stream of nitrogen at $60^{\circ} \mathrm{C}$. After the reconstitution with $3 \mathrm{~mL} 0.1 \mathrm{M}$ phosphate buffer $(\mathrm{pH}$ 6), the extracts were subjected to solid phase extraction and the eluates were analyzed by LCMSMS.

Separation was achieved on an Agilent 1200 HPLC System using Phenomenex Synergi $50 \times 2.0 \mathrm{~mm}$ Polar-RP column with $2.0 \mu \mathrm{m}$ particle size. Mobile phases used were A: $0.1 \%$ formic acid and B: acetonitrile with $0.1 \%$ formic acid. Separation was obtained using a linear gradient from $15 \%$ to $60 \%$ mobile phase B between 0.5 and 6.5 minutes and held for 0.1 minutes.

Analytes were detected on a Sciex 5500 Tandem Mass Spectrometer equipped with an electrospray ionization source in the positive ionization mode. Multiple reaction monitoring (MRM) was used for the following transitions: $\mathrm{m} / \mathrm{z} 342.22 \rightarrow$ $\mathrm{m} / \mathrm{z} 188.20$ for the internal standard fentanyl- $d_{5}, \mathrm{~m} / \mathrm{z} 337.15 \rightarrow \mathrm{m} / \mathrm{z} 187.90$ and 104.9 for fentanyl, $\mathrm{m} / \mathrm{z} 238.10 \rightarrow \mathrm{m} / \mathrm{z} 84.00$ for the internal standard norfentanyl- $d_{5}$, and $\mathrm{m} / \mathrm{z} 233.07 \rightarrow \mathrm{m} / \mathrm{z} 84.00$ and 150.10 for norfentanyl. For both fentanyl and norfentanyl, the lower limit of quantitation and limit of detection was $200 \mathrm{pg} / \mathrm{g}$ and $100 \mathrm{pg} / \mathrm{g}$, respectively. 
The $b / b_{0}$ was calculated for each umbilical cord specimen immunoassay result. The $b / b_{0}$ mean responses for the immunoassay results for the cases that received prenatal fentanyl and the cases that did not receive prenatal fentanyl were compared using the independent samples $t$ test to determine if there was a statistically different response for these two groups using our validated immunoassay procedure. Linear regression analysis was used to investigate the association between the $b / b_{0}$ results of the immunoassay responses and the total dose of fentanyl administered to the mothers prior to delivery. Independent samples t-test and linear regression were performed using IBM SPSS Statistics Subscription (updated November 30, 2020).

\section{Results}

The study included 62 subjects who met the inclusion criteria. Of these 62 subjects, 37 received fentanyl prior to delivery. The total amount of fentanyl administered ranged from $6.7 \mathrm{mg}$ up to $392 \mathrm{mg}$ (median, $74.9 \mathrm{mg}$ ). The total duration of fentanyl administration ranged from 0.5 hours to 80 hours (median, 47.4 hours) with the duration of fentanyl administration prior to birth ranging from 0.3 hours up to 11.7 hours (median, 3.7 hours).

Sixty-two (62) umbilical cord specimens were analyzed and only 1 umbilical cord specimen was reported positive using the previously described cutoffs. A strong immunoassay response for fentanyls was observed for this specimen $\left(\mathrm{b} / \mathrm{b}_{0}=10.6 \%\right)$ compared to the cutoff $\left(\mathrm{b} / \mathrm{b}_{0}=40.5 \% \pm 2.7 \%\right)$ and the measured concentrations, obtained using the LCMSMS confirmation procedure previously described, for fentanyl and norfentanyl were $6469 \mathrm{pg} / \mathrm{g}$ and $5241 \mathrm{pg} / \mathrm{g}$, respectively. Medical record review showed no documented outpatient prescription or in-hospital administration of fentanyl prior to delivery. This specimen also contained detectable quantities of amphetamine $(4 \mathrm{ng} / \mathrm{g})$, methamphetamine (19 $\mathrm{ng} / \mathrm{g})$, cotinine (128 ng/g), codeine (1 ng/g), morphine (21 ng/g), hydromorphone $(0.1 \mathrm{ng} / \mathrm{g})$ and 6-monoacetylmorphine (heroin metabolite; $0.1 \mathrm{ng} / \mathrm{g}$ ).

The remaining 61 umbilical cord specimens, including the 37 cases that received fentanyl prior to delivery, were analyzed by both methods. All specimen results were below the selected cutoffs utilized for the immunoassay initial test (500 pg/g) and the LCMSMS confirmation assay's limit of quantitation $(200 \mathrm{pg} / \mathrm{g})$.

Excluding the single screen and confirmed positive specimen, the mean $b / b_{0}$ for the cases that received fentanyl prior to delivery was $91.3 \% \pm 10.6 \%$ and the mean $\mathrm{b} / \mathrm{b}_{0}$ for the cases that did not receive fentanyl was $98.2 \% \pm 6.5 \%$. An independent samples $t$ test concluded that the observed mean $\mathrm{b} / \mathrm{b}_{0}$ difference (6.92; $95 \%$ CI: $2.54,11.30)$ was a statistically significant difference $[t(58.9)=3.158, p=$ 0.003]. The slope obtained using linear regression analysis of the total fentanyl delivered pre-delivery and $b / b_{0}$ of the fentanyl immunoassay test result for the corresponding umbilical specimen was $-0.087\left(\mathrm{R}^{2}=0.349\right.$; Figure 1$)$.

\section{Discussion}

Our study reports here for the first time a comparison of cases where fentanyl 


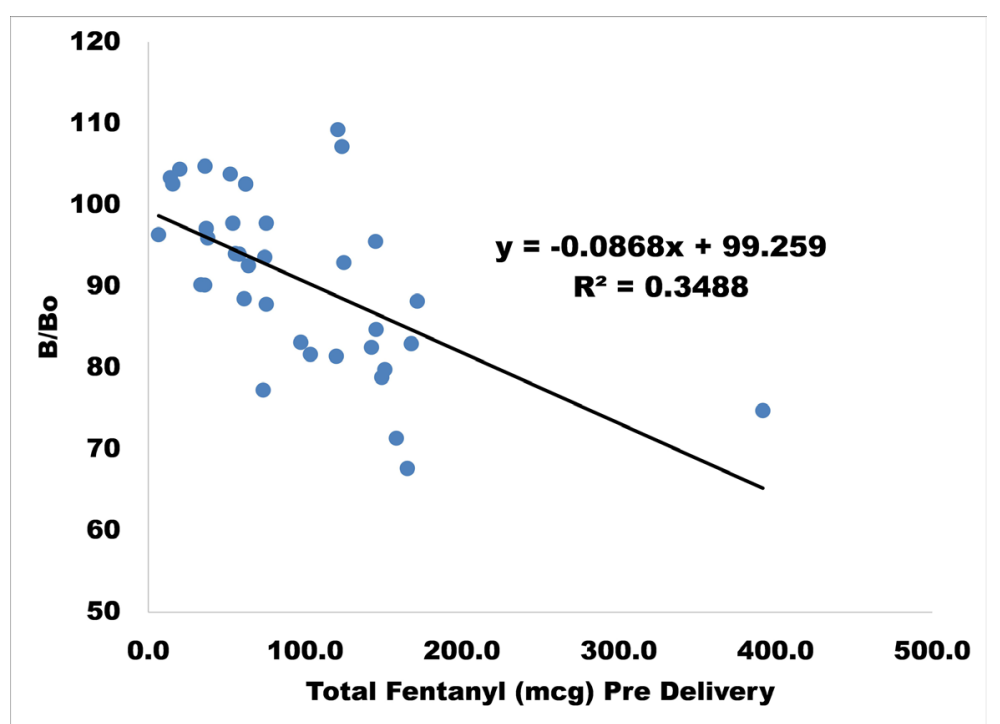

Figure 1. The slope obtained using linear regression analysis of the total fentanyl delivered pre-delivery and $\mathrm{b} / \mathrm{b}_{0}$ of the fentanyl immunoassay test result for the corresponding umbilical specimen was $-0.087\left(\mathrm{R}^{2}=0.349\right)$.

was administered or not administered by hospital staff to mothers prior to delivery using validated methods (immunoassay and LCMSMS) for the detection of fentanyl and its metabolite in umbilical cord tissue segments. Of the 62 umbilical cord specimens analyzed, only 1 of the specimens exceeded the arbitrarily selected cutoffs for both immunoassay and LCMSMS and this specimen also contained detectable amounts of amphetamine, methamphetamine, cotinine, codeine, morphine, hydromorphone, and 6-monoacetylmorphine.

Examination of the immunoassay responses of the means obtained for cases where the mother received fentanyl prior to delivery (mean $\mathrm{b} / \mathrm{b}_{0}=91.3 \% \pm$ $10.6 \%$ ) and for cases where there was not a record of receiving fentanyl (mean $\left.\mathrm{b} / \mathrm{b}_{0}=98.2 \% \pm 6.5 \%\right)$ found significant differences. Both groups specimen results were well removed from the immunoassay cutoff $\left(\mathrm{b} / \mathrm{b}_{0}=40.5 \% \pm 4.7 \%\right)$ and below LCMSMS confirmation assay's limit of quantitation $(200 \mathrm{pg} / \mathrm{g})$. Due to the specimens being below LCMSMS's limit of quantitation, we compared immunoassay $\mathrm{b} / \mathrm{b}_{0}$ values between cohorts rather than fentanyl levels (pg/g).

Further examination of the immunoassay responses compared to the total amount of fentanyl received for the cases where the record indicated the administration of fentanyl prior to delivery was conducted using linear regression. A slope of -0.087 was found indicating that for each $\mathrm{mg}$ of fentanyl received by the mother prior to delivery, we predict a decrease in the immunoassay $b / b_{0}$ of 0.087. The Pearson Product Moment was 0.349 which is consistent with a moderate association, with variation in the time between last fentanyl dose and delivery most likely limiting the correlation between fentanyl dose and $b / b_{0}$. These results preliminarily indicate a correlation of fentanyl dose to umbilical cord concentration, with concentrations increasing in umbilical cord with increasing dose administration. 
At this time, the experimental determination of an appropriate cutoff for fentanyl using umbilical cord tissue is lacking in the literature. It is desirable to use a cutoff that will detect illicit fentanyl while excluding cases where fentanyl was administered by hospital staff. Many jurisdictions are required to report positive newborn toxicology cases to the State and the resources required to document iatrogenic positives are wasteful of limited resources. Furthermore, atypical withdrawal symptoms have be described following prenatal fentanyl exposure, further highlighting the need for prompt, accurate identification of in utero exposure for optimized NAS management [6].

While the immunoassay procedure was able to distinguish between cases where fentanyl was administered and not administered, the responses were well removed from the cutoff. The single specimen where fentanyl and norfentanyl was reported above the cutoff came from a case where several other high-risk substances were reported (methamphetamine, heroin, and nicotine) and neither in-hospital or prescription fentanyl was documented. We determined that the initial testing cut off level of $500 \mathrm{pg} / \mathrm{g}$ was appropriate in differentiating signal from fentanyl administration during labor and illicit fentanyl. Additionally, this study provided evidence for potential future cutoff lowering while still avoiding the inconvenience of detecting hospital-administered fentanyl.

This is the first study appearing in the literature that compares umbilical cord testing results for maternal cases where fentanyl was administered or not administered by hospital staff. However, there are several limitations to the study. This study is a retrospective analysis of a convenience sampling coming from a single hospital which limits the generalizability of the findings. No records were found of outpatient fentanyl prescriptions, however prescriptions from outside CAMC that were undisclosed to staff may have been missed. Additionally, due to the small sample size, only one instance of illicit fentanyl use was discovered. A prospective random controlled trial is not feasible due to ethical reasons. More studies are needed to confirm the adequacy of the selected cutoff and to determine long-term outcomes in infants exposed to fentanyl in utero.

\section{Conclusion}

This project is one small part of ongoing efforts to combat the opioid epidemic that has gripped our society over the past several years. This study demonstrated that umbilical tissue is a suitable specimen type for the detection of fentanyl. Additionally, this study has demonstrated that the cutoffs selected appear to adequately identify illicit fentanyl use while not flagging cases where fentanyl was administered by the hospital prior to birth.

\section{Conflicts of Interest}

Stefan Maxwell is an employee of Pediatrix Medical Group (a subsidiary of MEDNAX) who is contracted with Charleston Area Medical Center to serve as their NICU medical director. Dr. Maxell has no conflicts of interest to declare. 
Ryan Mitacek and Stephanie Thompson are employees of Charleston Area Medical Center and have no conflicts of interest to declare. Joseph Jones and Donna Coy are employees of United States Drug Testing Laboratories. United States Drug Testing Laboratories is in the business of selling the tests described in this manuscript. This relationship has not in any way influenced the direction or results of this study.

\section{Author Contributions}

Joseph Jones and Ryan Mitacek developed the study design. Stephanie Thompson led the efforts to obtain Institutional Review Board approval. Stefan Maxwell served as the principal investigator for this study. Ryan Mitacek conducted the review of the medical records. Joseph Jones and Donna Coy reviewed and analyzed the toxicology data. All five authors participated in the composition and review of the manuscript.

\section{References}

[1] Hudak, M.L. and Tan, R.C. (2012) Neonatal Drug Withdrawal. Pediatrics, 129, e540-e560. https://doi.org/10.1542/peds.2011-3212

[2] McQueen, K. and Murphy-Oikonen, J. (2016) Neonatal Abstinence Syndrome. The New England Journal of Medicine, 375, 2468-2479. https://doi.org/10.1056/NEJMra1600879

[3] Patrick, S.W., Schumacher, R.E., Benneyworth, B.D., Krans, E.E., McAllister, J.M. and Davis, M.M. (2012) Neonatal Abstinence Syndrome and Associated Health Care Expenditures: United States, 2000-2009. JAMA, 307, 1934-1940. https://doi.org/10.1001/jama.2012.3951

[4] Winkelman, T., Villapiano, N., Kozhimannil, K.B., Davis, M.M. and Patrick, S.W. (2018) Incidence and Costs of Neonatal Abstinence Syndrome among Infants with Medicaid: 2004-2014. Pediatrics, 141, e20173520.

https://doi.org/10.1542/peds.2017-3520

[5] Kee, W.D.N., Khaw, K.S., Ng, F.F., Ng, K.K., So, R. and Lee, A. (2014) Synergistic Interaction between Fentanyl and Bupivacaine Given Intrathecally for Labor Analgesia. Anesthesiology, 120, 1126-1136. https://doi.org/10.1097/ALN.0000000000000118

[6] Nellhaus, E.M., Murray, S., Hansen, Z., Loudin, S. and Davies, T.H. (2019) Novel Withdrawal Symptoms of a Neonate Prenatally Exposed to a Fentanyl Analog. Journal of Pediatric Health Care, 33, 102-106. https://doi.org/10.1016/j.pedhc.2018.08.014

[7] Wong, C.A. (2009) Advances in Labor Analgesia. International Journal of Women's Health, 1, 139-154. https://doi.org/10.2147/IJWH.S4553

[8] Montgomery, D., Plate, C., Alder, S.C., Jones, M., Jones, J. and Christensen, R.D. (2006) Testing for Fetal Exposure to Illicit Drugs Using Umbilical Cord Tissue Vs meconium. Journal of Perinatology, 26, 11-14. https://doi.org/10.1038/sj.jp.7211416

[9] Jones, J.T., Jones, M., Jones, B., Sulaiman, K., Plate, C. and Lewis, D. (2015) Detection of Codeine, Morphine, 6-Monoacetylmorphine, and Meconin in Human Umbilical Cord Tissue: Method Validation and Evidence of in Utero Heroin Exposure. Therapeutic Drug Monitoring, 37, 45-52.

https://doi.org/10.1097/FTD.0000000000000104 
[10] Marin, S.J., Metcalf, A., Krasowski, M.D., Linert, B.S., Clark, C.J., Strathmann, F.G., et al. (2014) Detection of Neonatal Drug Exposure Using Umbilical Cord Tissue and Liquid Chromatography Time-of-Flight Mass Spectrometry. Therapeutic Drug Monitoring, 36, 119-124. https://doi.org/10.1097/FTD.0b013e3182a0d18c 